\title{
TOWARDS CONTINUOUS MONITORING OF ARCHITECTURE. TERRESTRIAL LASER SCANNING AND MOBILE MAPPING SYSTEM FOR THE DIAGNOSTIC PHASES OF THE CULTURAL HERITAGE
}

\author{
M. Campi ${ }^{1}$, M. Falcone ${ }^{2}$, S. Sabbatini ${ }^{3}$ \\ ${ }^{1}$ University of Naples ''Federico II' - Dep. of Architecture, campi@ unina.it \\ ${ }^{2}$ University of Naples 'Federico II' - Dep. of Architecture, marika.falcone@unina.it \\ 3 3Dscan - Smart Surveying, ss@3dscan.it
}

Commission II

\begin{abstract}
In recent decades, the significant advances in digital technologies applied to cultural heritage have led to profound transformations in the activities of protection, conservation and enhancement. A current field of experimentation concerns 'rapid mapping' survey techniques with mobile mapping system (MMS). The use of these methodologies is increasingly widespread for the diagnostic and monitoring phases of the architectures. The potential of these techniques are manifold: these systems record, in a short time, a large number of information with a rather high level of accuracy. In particular, the research presented here has studied an 'innovative' Lidar mobile mapping methodology, NavVis VLX, with experimental perspectives, for the survey of the Cripta of the Cathedral of San Matteo in Salerno (Italy).
\end{abstract}

KEY WORDS: Mobile Mapping System, Terrestrial Laser scanning, Data processing, Monitoring, Comparison

\section{INTRODUCTION}

In recent decades, the significant advances in digital technologies applied to architectural and cultural heritage in general have led to profound transformations in the activities of protection, conservation and enhancement. The introduction of the new application methodologies, assisted by advanced equipment and procedures, have, in fact, marked the beginning of a new era that nowadays lives on new knowledge dissemination platforms that develop, at the same time, new ways of transmitting the knowledge. In the contemporary scenario, these precepts radically change the approach to the conservation of architecture by introducing the dissemination and construction of predictive models in the preliminary diagnostic investigation of the restoration project. In this framework, the survey discipline is configured as the main element of the experimentation and the evaluation of the methods and equipment for the acquisition phases, leads to considerations relating to the investigation procedures and the return of dimensional, colorimetric and morphological data. In fact, a current field of experimentation concerns 'rapid mapping' survey techniques with mobile mapping system acquisition systems. For this reason, the use of these methodologies is increasingly widespread for the diagnostic and monitoring phases of the Cultural Heritage. The potential of these techniques is manifold: these systems record, in a short time, a large amount of information with a rather high level of accuracy. In particular, the research presented here has applied an 'innovative' Lidar mobile mapping methodology, NavVis VLX, with experimental perspectives, for the survey of the Cripta of the Cathedral of San Matteo in Salerno (Italy).

\section{RELATED WORKS}

In this constantly evolving scenario, the introduction of new methods of documentation and recording of the morphometric characteristics, through the use of MMS survey methodologies, have strongly influenced the activities related to the monitoring of cultural heritage that require a continuous collection of threedimensional data.

The use of these devices constitutes a real innovation in the Geomatics sector and the undisputed value of mobile mapping systems is the possibility of significantly accelerating the data acquisition and processing phases (Bonfanti, 2021). The researches on the subject are manifold and show a variety of hardware solutions, with dynamic acquisition procedures through the use of trolleys, backpacks, trolleys, and software. The three hardware components of an MMS system are optical sensors, navigation and positioning sensors (IMU / GNSS) and a control and synchronization unit. The absence of GNSS signal and positioning data in the case of indoor surveys has implemented the development of new methods. These methods are based on an overlap of data using an inertial platform (Inertial Navigation System - IMU) and a "Simoultaneous Localization and Mapping" (SLAM) algorithm for the definition of the trajectory and the three-dimensional reconstruction of the recorded scenes (Lehtola et al., 2017).

The state of the literature highlights how the most up-to-date studies are focusing on the validation of these new instruments by comparing them with consolidated acquisition techniques based on active sensors, such as terrestrial laser scanning (TLS) and passive sensors. (Toschi et al., 2015; Nocerino et al., 2017; Nespeca, 2018, Sammartano et al., 2018; Di Stefano et al., 2020; Kaldova et al., 2020; Campi et al., 2021; Pulcrano et al., 2020; al., 2021).

In the last decade, the Tir Lab research group and the 3DSCAN company have also had different experiences in this field. In this regard, the research carried out by the Tir Lab Laboratory in 2009 where, for the first time, the Optech Lynx system integrated with the Applanix POS-LV 420 system was used for the urban survey of the area in front of Piazza Plebiscito and the Teatro di San Carlo in Naples (Campi, 2009) and in 2017 for the architectural survey of the Complex of Monte Sant'Angelo in Naples where the first comparisons between static and dynamic acquisition systems were presented (Campi et al., 2018). 
These researches were a useful reference for preparing the operational phases of the survey reported by analyzing the case studies that saw the use of mobile mapping system (MMS) and static systems such as Laser Scanner (TLS).

The experiment, presented here, is part of a scientific collaboration agreement established between the Department of Architecture of the University of Naples " Federico II " Laboratorio Tir Lab, coordinated by professors A. di Luggo and M. Campi and the 3DSCAN-SmartSurvey company.

Furthermore, this contribution is part of a broader and still ongoing research line, within the framework of the $\mathrm{PhD}$ in Architecture at the aforementioned university $(\mathrm{PhD}$ candidate $\mathrm{M}$. Falcone - tutor M. Campi and co-tutor S. Di Martino).

\section{CASE STUDY}

The investigation was conducted, in particular, on the case study of the Crypt of the Cathedral of San Matteo, located in the historic center of the city of Salerno (Italy). The object of the analysis carried out was chosen for its intrinsic characteristics: the environment, in fact, is hypogeum with a unique architectural and artistic conformation. The space, marked by columns and with the apses in correspondence with those of the upper transept, represents an absolutely novel element inside the Cathedral (fig. $1)$.

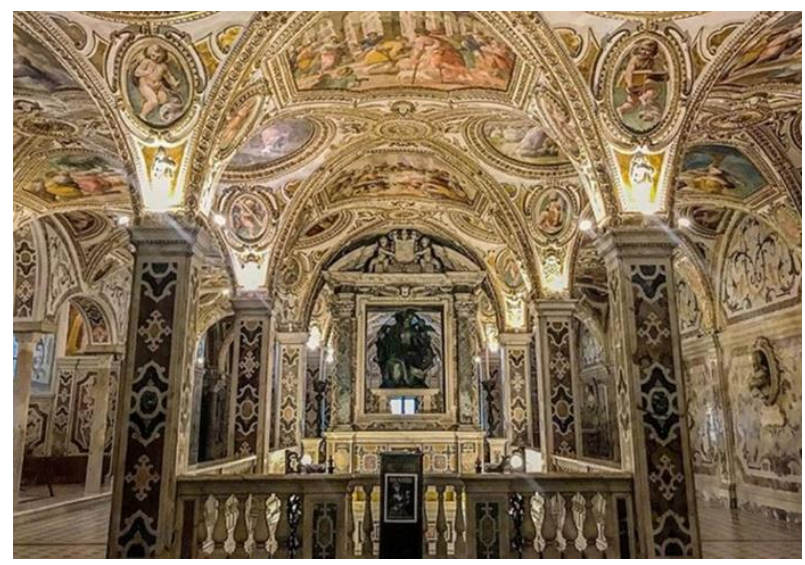

Figure 1. The Cripta of the Cathedral of San Matteo in Salerno (Italy)

Its structure "ad aula", constitutes the first example in all of Central and Southern Italy, in fact, this architecture "well known in Europe and Northern Italy, was absolutely unusual in Southern Italy" (Braca, 2018). According to the legend, the saint's spolias were brought to Salerno by Gisulfo I in the 10th century and when the new cathedral dedicated to the evangelist was built, they were placed in the Crypt. The crypt is divided into three parts by a double row of columns, which have the specific function, together with the others located at the corners and inside the apses, to support the upper transept. The spatiality is to be found in the main lines still corresponding to the original ones of the medieval era, even if the major decorative changes occurred starting from the seventeenth century. Instead, the main spatial transformation took place in the fifties of the last century when the staircase leading to the tomb of St. Matthew was built, distorting the architectural structure of the medieval era. The crypt, as seen today, however, corresponds to the works carried out in the early seventeenth century when the viceroy of Naples Fernando Ruiz de Castro VI, by the will of the Spanish kings Philip II and Philip III, financed the intervention in the crypt of Salerno. Along the walls there are ten half-length marble sculptures inside oculi, depicting the first bishops of Salerno, nine of which were made by Francesco Ragozzino in 1759. The tenth was a work of the sixteenth century and depicts St. John the Baptist. In the left apse, called "delle Santi Martiri", on the altar there is a painting of the naturalistic school depicting "la Madonna con il bambino" and the holy martyrs. In the other minor apse, known as the " $i$ Santi Confessori", there is a painting depicting the bishops, of late mannerist invoice, but updated on naturalistic canons. Instead, through various archival and bibliographic sources it can be deduced that the space organized around the tomb of San Matteo was the real beating heart, not only of the Cathedral, but of the entire city of Salerno.

\section{DEVELOPED METHODOLOGY}

To conduct a diagnostic investigation in preparation for the construction of outputs such as forecasting models for restoration, it was necessary to develop a working methodology divided into three phases and the choice of technologies and investigation methods used was necessary to answer the research question fixed.

The first phase relates to the bibliographic and archival investigation of the site which made it possible to reconstruct the historical events and transformations that have taken place over the centuries. The historical and artistic importance of the hypogeum environment and the very rich decorative apparatus inside, required a particular accuracy of the data and, at the same time, a remarkable speed of acquisition, due to short times due to the high flow of visitors. Therefore, the survey activities, which were configured in the second phase, were planned with a view to obtaining a three-dimensional data with high metric and geometric detail, capable of ensuring an appropriate display of the morphology of the spaces analyzed.

After evaluating the architectural characteristics of the site and the numerous problems encountered within it, acquisition and processing procedures were used for the collection of morphometric data, which saw the use and integration of two different range-based methodologies : (i) TLS Terrestrial Laser Scanner phase modulation survey with Focus 3D s120 (ii) mobile mapping survey with Navvis VLX, a SLAM wearable mapping device equipped with two Lidar sensors and four cameras positioned on the top of the device with full acquisition $360^{\circ}$ (fig. 2);

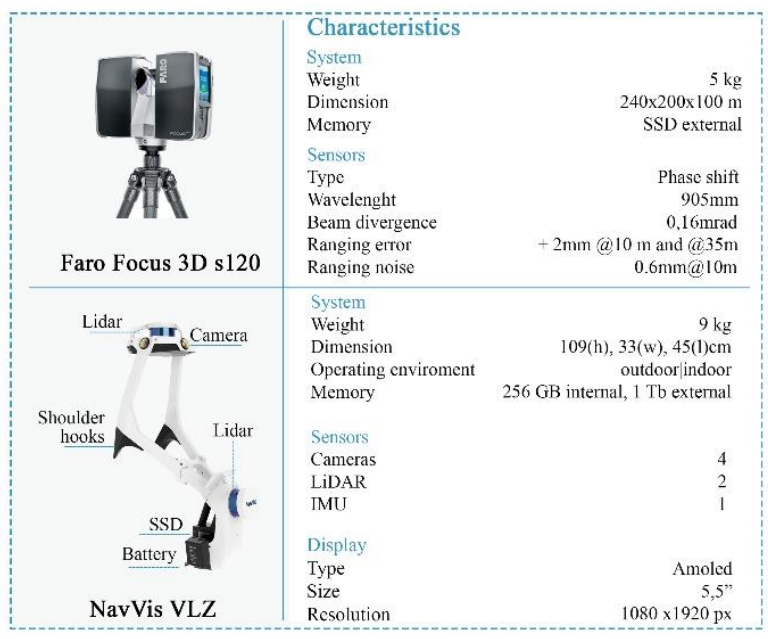

Figure 2. The characteristics of the range-based technologies used

Finally, in the third and last phase, the comparison of the threedimensional models obtained made it possible to highlight the 
differences in the data in terms of quantity and quality of points processed by different sensors, as well as the results deriving from the static and dynamic acquisition.

\subsection{Static acquisition: Surveys with phase modulation sensor}

Based on the above considerations, for the first acquisition campaign, the survey of the Crypt of San Matteo was performed with a phase modulation laser sensor Faro Focus 3D s120 supplied to the Tir Lab Laboratory of the Department of Architecture of the University of Studies. of Naples " Federico II ". The sensor used returns nominal distance information in a range between $0.60 \mathrm{~m}$ and $120 \mathrm{~m}$, with an accuracy of $\pm 2 \mathrm{~mm}$ at 25 meters and an angular resolution of $0.009^{\circ}$. The field of view on the horizontal axis is $360^{\circ}$ while the vertical one is $305^{\circ}$ with an acquisition speed of 976,000 points / sec.

Furthermore, the instrumentation has a weight of about $5 \mathrm{Kg}$ (with dimensions equal to $240 \times 200 \times 100 \mathrm{~m}$ ) which allows a quick use in the field with a set up on a photographic tripod. Furthermore, a digital camera with an optical axis coaxial to the laser measurement beam is integrated in the instrument, which allows the restitution of the color information of each recorded point through the RGB values. Having examined the technical characteristics of the instrumentation examined, we proceeded to the data acquisition phases. After analyzing the morphology of the area under study, the positions of scans useful to cover the entire complex of the Crypt were identified and, in this case, it was essential to adopt strategies that reduced the number of acquisitions to a minimum, optimizing time in situ and avoiding the acquisition of a multitude of data difficult to manage during the processing phase. Therefore, the scanning stations were placed along the double row of counting columns and the entire area was surveyed through the acquisition of 6 range maps, setting an average resolution of $6 \mathrm{~mm}$ at a distance of 10 meters and a $4 \mathrm{x}$ accuracy.

In order to facilitate the subsequent processing and alignment phases of the individual range maps, spherical targets and planes were used for the referencing of the individual clouds. In this way, it was possible to ensure a high definition of the scanned surfaces, through adequate overlapping between clouds of consecutive points and, at the same time, the difficulties in managing the file due to data redundancy were limited. The settings of the parameters chosen influenced the data acquisition time and this phase was conducted in the field by two operators and ended in about 2 hours of work (fig. 3 ).
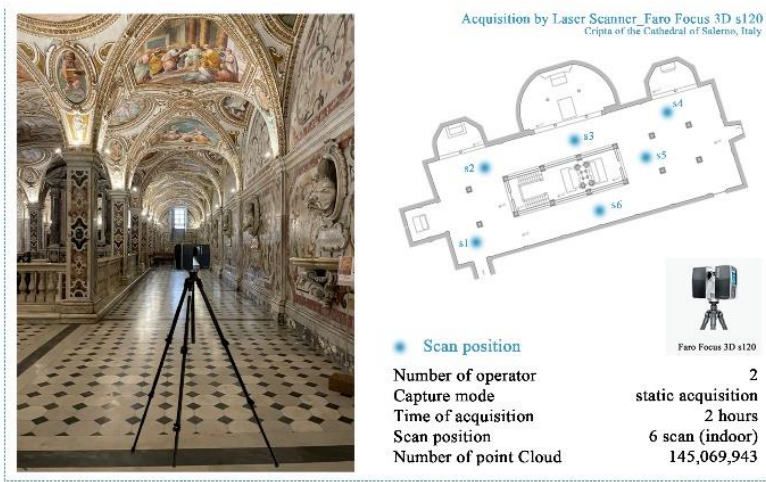

Figure 3. Acquisition by Laser Scanner-Faro Focus 3D s120: route scheme and parameters used

Once the survey operations in situ were completed, the data processing phases were carried out. The 6 range maps were aligned with the proprietary software Faro Scene and this processing consisted in the construction of the roto-translation matrix of the single scans, carried out by identifying three common points or two common points using the inclinometer between adjacent scans.

In the alignment phases, semi-automatic recognition procedures of targets and natural points were used, which returned a final cloud of approximately 145,069,943 points. This procedure recorded levels of alignment error of less than $2 \mathrm{~mm}$ in the vicinity of the instrument and of $4 \mathrm{~mm}$ for areas with greater distances (fig. 4).

Subsequently, the point cloud was imported into Autodesk's Recap Pro application for data management. More precisely, the cloud has been cleaned up by eliminating the noise.

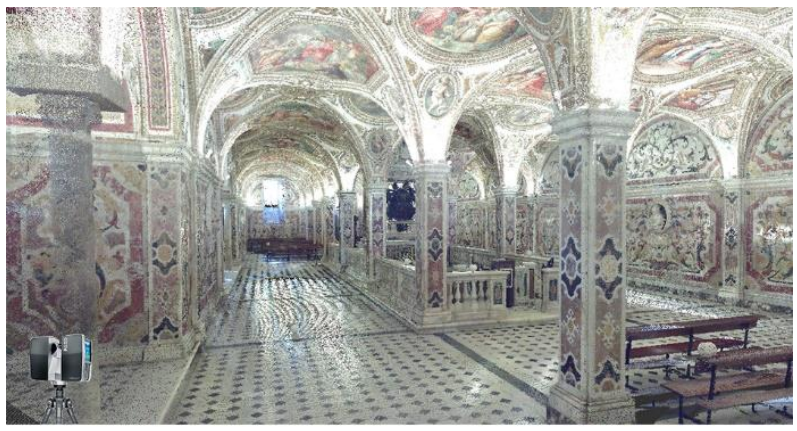

Figure 4. Point cloud acquired by Faro Focus 3D s120 and processed in the proprietary software Faro Scene

\subsection{Dynamic acquisition: Lidar mobile mapping survey with NavVis VLX}

The second phase of data acquisition examined a dynamic scanning procedure methodology. In particular, the entire Crypt was surveyed with a NavVis VLX mobile mapping system - from the German company NavVis.

This type of instrumentation allows you to detect threedimensional information by wearing the instrument and walking inside the Crypt. It is composed of two LiDAR sensors, positioned at the chest and head, which record threedimensional measurements in which the colorimetric data is integrated by four cameras positioned on the upper part of the device with high resolution $360^{\circ}$ image acquisition. Everything is recorded in real-time, showing the path taken on the interface, thus ensuring that the detected data can be verified. The instrumentation, with dimensions $109 \times 33 \times 56$ $\mathrm{cm}$, has a weight of about $9 \mathrm{~kg}$ (including the weight of the batteries) and is able to acquire up to 600,000 points per second with a maximum distance of 40 meters in indoor environments and 30 meters in outdoor environments and an accuracy of about $6 \mathrm{~mm}$. The ability to record data on the go, together with an accurate planning phase to eliminate drift errors, made field work extremely fast. In fact, it is important to underline, as in the aforementioned case, the acquisition times of the underground environment have drastically reduced compared to the laser scanner survey.

The acquisition lasted 11 minutes of work and the threedimensional data were recorded in a single loop while walking, with a constant step in the Crypt. To reduce possible divergences, the "round-trip" technique was used in the acquisition phases, ending the acquisition at the starting point. In this phase, no targets were used and the survey was conducted by a single operator. In parallel to the scan, however, the operator took numerous spherical photos in manual mode with an interval of about one and a half meters (fig. 5). 

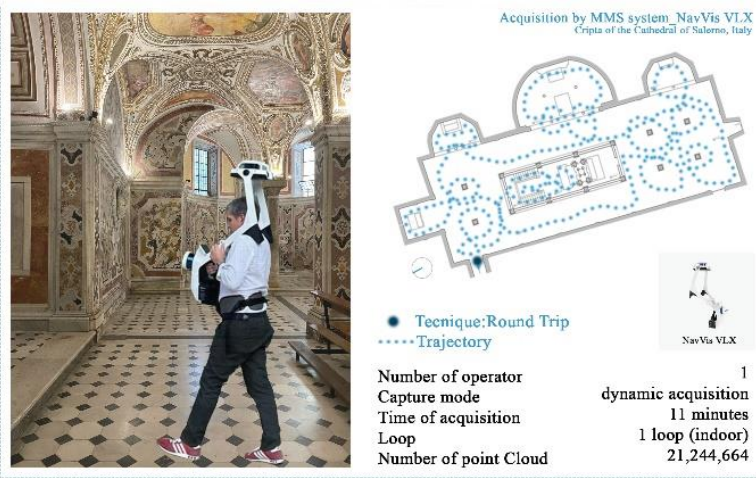

Figure 5. Acquisition by MMS system_NavVis VLX route scheme and parameters used

At the end of the acquisition phase, the processing of the data coming from the active sensor took place through the cloud platform of the NavVis IVION proprietary software. The result is a cloud of $21,244,664$ points (fig. 6).

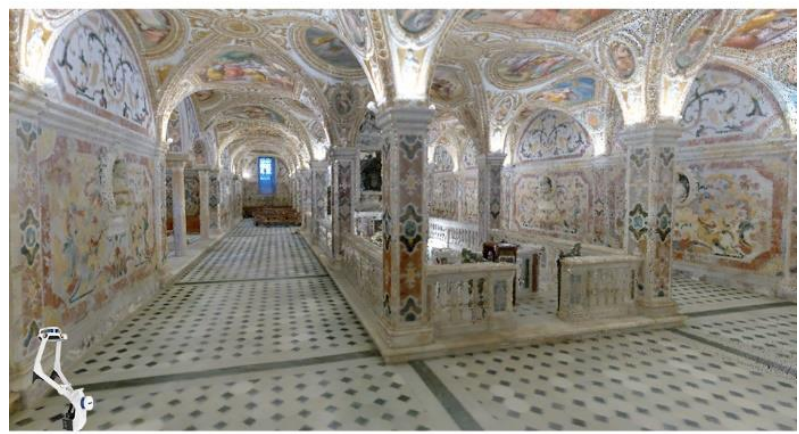

Figure 6. Point cloud acquired by NavVis VLX and processed in NavVis IVION

\section{ANALYSIS E COMPARISON}

After the three-dimensional data processing phases, the results obtained constituted a fundamental support for the analysis of the site. These results were examined, with the comparison of threedimensional models, at different scales of detail, from the underground environment as a whole to the decorative apparatus. Further reflections were also conducted on the accuracy and speed of data acquisition and processing, obtained from two different survey methods, which produced two different point clouds.

Thus, it was possible to compare the geometric accuracy of the point clouds by evaluating the limits and potential of each methodology with subsequent punctual analyzes on the roughness values.

The results of the comparisons made are shown below.

A first phase of evaluation, carried out by the authors, provided for a direct observation of the two-point clouds that show overall a good three-dimensional reconstruction of the Crypt, with the exception of some areas.

The first obvious difference is in the quality of the data collected. The data, detected by the Faro 3D s120 laser scanner, are more detailed than those of the NavVis VLX instrumentation, while the colorimetric characteristics of the latter are undoubtedly superior. Upon closer visual observation, the NavVis point cloud is complete in all its parts unlike the point cloud of Laser scanner which shows, due to the static acquisition of the instrument some shortcomings in the vicinity of the vaulted rooms of the apses (fig. 7).

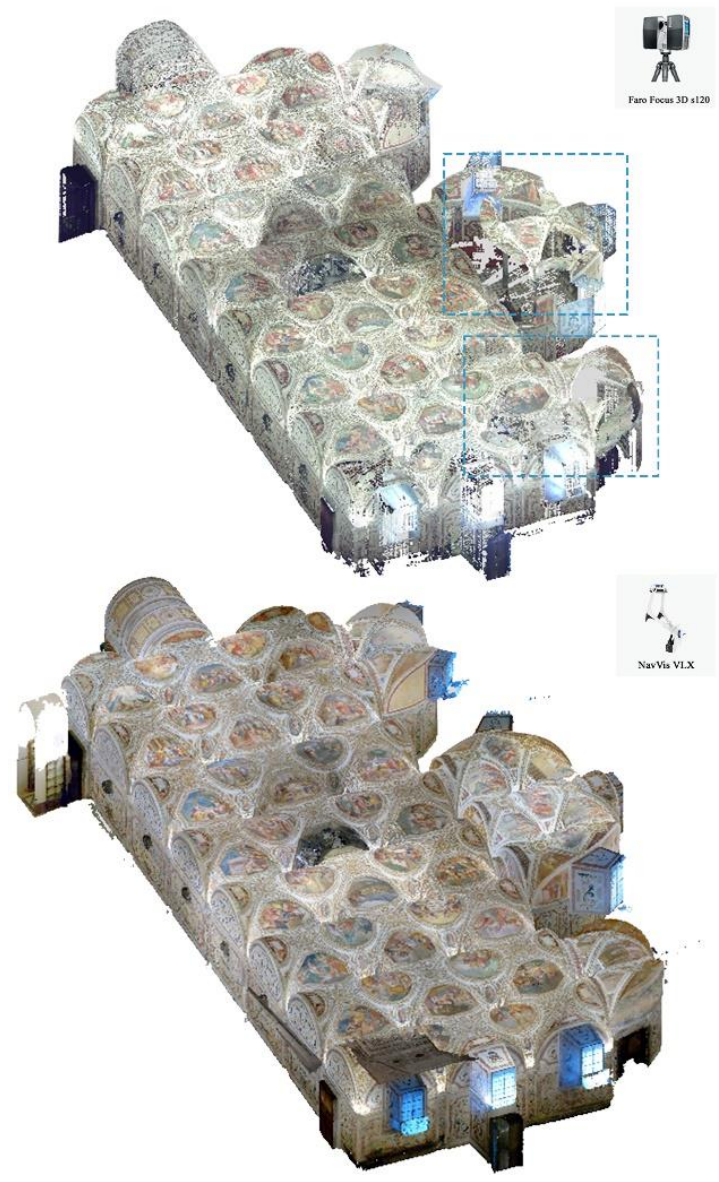

Figure 7. Above the point cloud acquired by the laser scanner below the point cloud acquired by the NavVis VLX

Before proceeding to the first comparison, it was necessary to filter the data by decimating the cloud generated by the laser scanner instrumentation which counted a number of points significantly higher than the cloud acquired with the MMS method.

Subsequently, the alignment procedures were carried out in the open source application Cloud Compare where it was necessary to align the NavVis VLX point cloud to the Laser scanner dataset, used as a reference cloud for the registration procedure with the ICP algorithm (Interactive Closest Point). For the alignment procedure, five homologous points, characteristic of the two models, were manually identified. At this point a rigid rototranslation was applied, finding an RMSE (Root Mean Square Error) alignment error between the two data datasets equal to $0.03 \mathrm{~m}$. Finally, the cloud / cloud distance values were calculated. This first comparison, as shown in Figure 8, shows absolutely satisfactory results. As we can read in the histogram, the distribution of the points analyzed shows that most of them fall within the error range between 0 and $0.025 \mathrm{~m}$. The parts in red highlight the values of greater distance (fig. 8).

Furthermore, the integration of the data allowed a reading of the hypogeum environment from which the information useful for the two-dimensional graphic representation was extracted through horizontal and vertical section planes (fig. 9). 


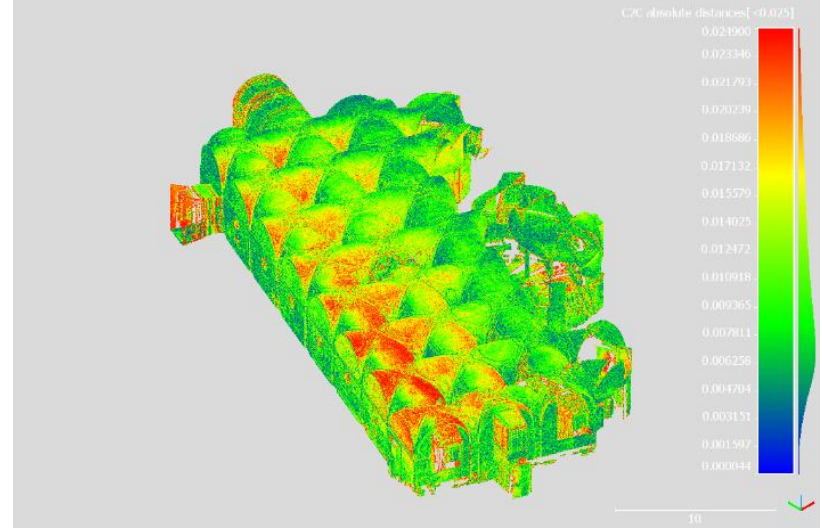

Figure 8. Comparison in Cloude Compare. Analysis cloud2cloud (c2c) distance
Wanting to target a field of experimentation that concerns 'rapid mapping' survey techniques for the continuous monitoring of architectures with mobile mapping system acquisition systems, in this preliminary phase of the research, further analyzes were conducted, focused on the evaluation of each portion of the two acquired datasets. Therefore, the two datasets were compared again by analyzing the noise level, using the "roughness" (sphere $\mathrm{r}=0.025 \mathrm{~m})$ mathematical algorithm implemented in CloudCompare. The areas considered were two: a portion of the intrados of the vault characterized by a strong decorative apparatus and placed in correspondence with the staircase leading to the Tomb of the Holy Matthew and the half-length marble statue inside the oculus depicting the saint "San Giovanni Battista". The first analysis was carried out on a portion of the intrados of the vault. In comparison, this type of analysis reported the following results: the point cloud of the laser scanner dataset is characterized by a noise level of 0.004 meters with a homogeneous of the points and a higher level of accuracy of the
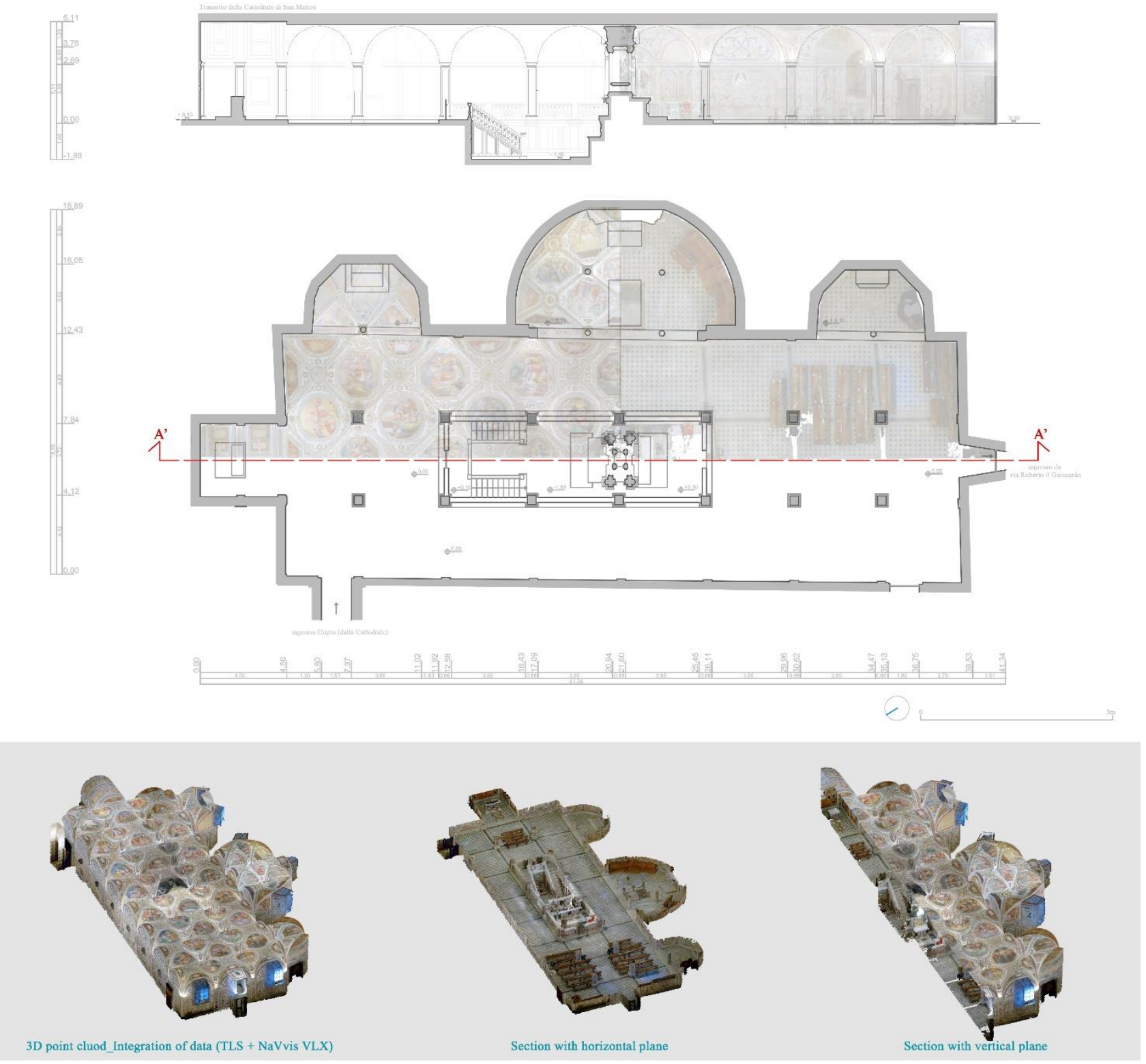

Figure 9. Graphic elaboration based on 3D data acquired 
decorative apparatus while the cloud of points NavVis VLX has slightly lower noise values which are around 0.0035 meters with a sparser distribution of points but which in any case do not allow easy reading of geometric and detailed information (fig. 10).
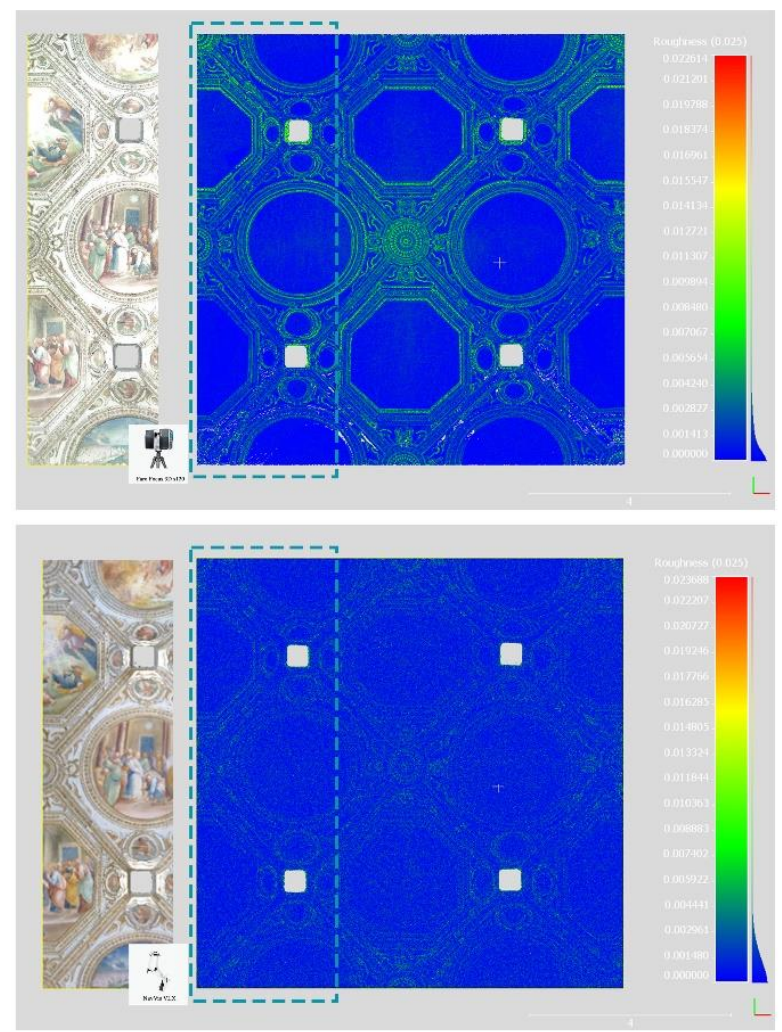

Figure 10. Roughness comparison of the vault between TLS (above) and MMS (down). The analysis has been performed using CC (sphere $\mathrm{r}=0.025 \mathrm{~m}$ ).

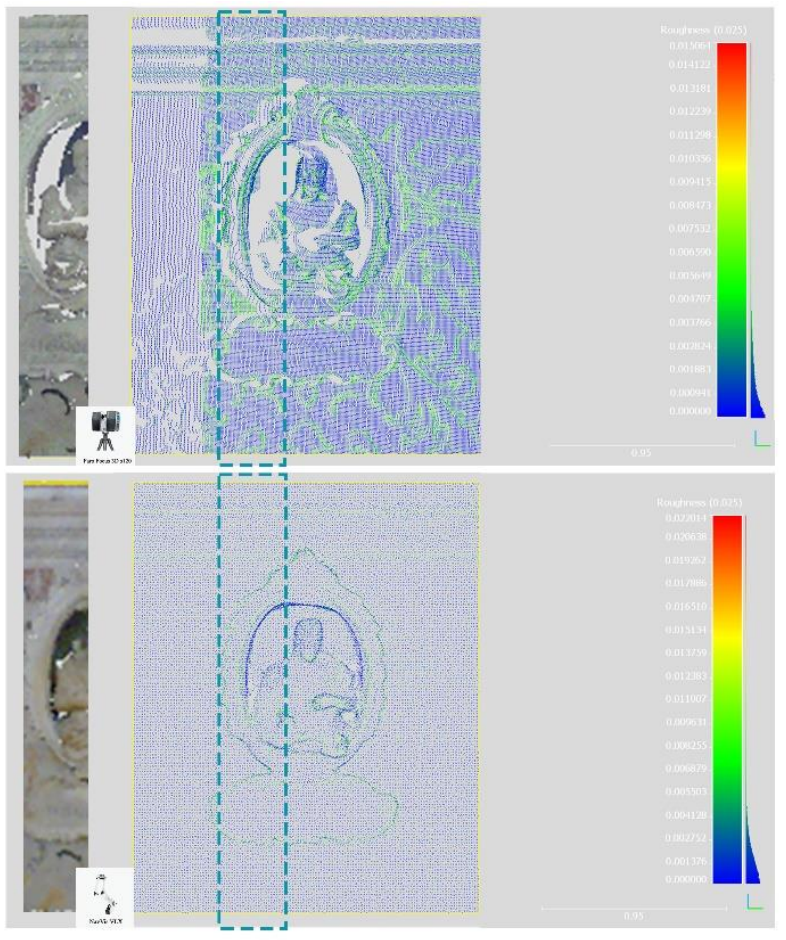

Figure 11. Roughness comparison of the marble statue beetween TLS (above) and MMS (down). The analysis has been performed using CC (sphere $\mathrm{r}=0.025 \mathrm{~m})$.
Subsequently we proceeded to the second and final analysis on the marble statue.

For the analysis of the noise of the statue, values equal to 0.0027 $\mathrm{m}$ for the NavVis cloud and $0.0014 \mathrm{~m}$ for the laser scanner cloud were found. (Figure 11);

The NavVis VLX cloud has a higher noise level and for this value it is necessary to take into account the dynamic acquisition technique which has allowed to record a greater number of threedimensional data compared to the static type acquisition which, in fact, presents a cloud sparse and with some missing parts.

\section{CONCLUSIONS}

In conclusion, the study aimed at continuous monitoring of the architectures examined a methodology with MMS dynamic scanning compared with a consolidated TLS static scanning system. As can be seen from the analyzes carried out, the results of the experimentation are satisfactory. In this case, the reduced times of the acquisition phases together with the accuracy of the dimensional and colorimetric data make the NavVis VLX instrumentation a powerful means for "rapid mapping" survey, opening up, at the same time, new challenges in the field of Cultural Heritage.

\section{ACKNOWLEDGEMENTS}

The authors strongly wish to thank all those who participated and collaborated in the project. We wish to thank Don Michele Pecoraro, parish of the Cathedral of SS. Matteo andGregorio di Salerno (Italy), who made it possible to carry out the surveys inside the Crypt and the entire complex. Special thanks to the entire team of the company and in particular to Francesco Sala, co-CEO of the 3DSCANSmartSurvey company for the support and constant help during the three-dimensional data processing phases. Special thanks to arch. Valeria Cera, member of the Tir Lab Laboratory, coordinated by professors Antonella di Luggo and Massimiliano Campi, at the Department of Architecture of the University of Naples Federico II, which supported the TLS survey operations. Finally, the authors would like to thank Chiara Melandri, of the NavVis company.

\section{REFERENCES}

Braca A., 2018. Guida illustrata alla Cattedrale di San Matteo. Salerno: Opera edizioni, pp.105-109. ISBN 88-88773-71-1.

Bonfanti, C., Patrucco, G., Perri, S., Sammartano, G., and Spanò, A., 2021. A new indoor lidar-based mms challenging complex architectural environments, int. Arch. Photogramm. Remote sens. Spatial inf. Sci., xlvi-m-1-2021, 79-86, https://doi.org/10.5194/isprs-archives-xlvi-m-1-2021-79-2021.

Campi, M., di Luggo, A., Monaco, S., Siconolfi, M., and Palomba, D., 2018. Indoor and outdoor mobile mapping systems for architectural surveys, Int. Arch. Photogramm. Remote Sens. Spatial Inf. Sci., XLII-2, 201-208, https://doi.org/10.5194/isprsarchives-XLII-2-201-2018.

Campi, M., 2009. La città specchiata: percorsi di rilievo urbano. Tecniche di scansione laser da piattaforma mobile in Atti di convegno, Università degli Studi di Napoli ''Federico II'.

Campi, M., di Luggo, A., and Falcone, M., 2021. Photogrammetric processes and augmented reality applications using mobile devices, Int. Arch. Photogramm. Remote Sens. Spatial Inf. Sci., XLVI-M-1-2021, 101-106, 
https://doi.org/10.5194/isprs-archives-XLVI-M-1-2021-101-

2021.

Di Stefano F., Cabrelles M., Garcia-Asenjo L., Lerma JL, Malinverni ES., Baselga S., Garrigues P., Pierdicca R., 2020. Evaluation of long-range mobile mapping system (MMS) and close-range photogrammetry for deformation monitoring. A case study of cortes de pallas in Valencia (Spain). Applied Sciences (Switzerland). 10(19):6831.

Kalvoda, P., Nosek, J., Kuruc, M., Volarik, T., Kalvodova, P., 2020. Accuracy Evaluation and Comparison of Mobile Laser Scanning and Mobile Photogrammetry Data in IOP Conf. Ser.: Earth Environ. Sci. 609012091.

Lehtola, V.V.; Kaartinen, H.; Nüchter, A.; Kaijaluoto, R.; Kukko, A.; Litkey, P.; Honkavaara, E.; Rosnell, T.; Vaaja, M.T.; Virtanen, J.-P.; Kurkela, M.; El Issaoui, A.; Zhu, L.; Jaakkola, A.; Hyyppä, J., 2017. Comparison of the Selected State-Of-TheArt 3D. Indoor Scanning and Point Cloud Generation Methods. Remote Sens. $\quad$ 9, 796, https://doi.org/10.3390/rs9080796.

Nespeca, R., 2018. Towards a 3D digital model for management and fruition of Ducal Palace at Urbino. An integrated survey with mobile mapping. SCIRES-IT- SCIentific RESearch and Information Technology, 8(2), 1-14. https://doi.org/10.2423/I22394303V8N2P1.

Nocerino, E., Menna, F., Remondino, F. Toschi, I. and Rodriguez-Gonzalvez, P.: "Investigation of indoor and outdoor performance of two "portable mobile mapping systems," in Videometrics, Range Imaging, and Applications XIV, vol. 10332, p. 103320I. International Society for Optics and Photonics.

Pulcrano, M., Scandurra, S., Fragalà, E., Palomba, D., and di Luggo, A.,2021. Multi-sensor data acquisition and integration processes for the study and documentation of the church of Santa Maria degli Angeli in Pizzofalcone in Naples, Int. Arch. Photogramm. Remote Sens. Spatial Inf. Sci., XLVI-M-1-2021, 571-578, https://doi.org/10.5194/isprs-archives-XLVI-M-12021-571-2021.

Sammartano, G., \& Spanò, A., 2018. Point clouds by SLAMbased mobile mapping systems: accuracy and geometric content validation in multisensor survey and stand-alone acquisition. Applied Geomatics, 10(4), 317-339.

Toschi, I., Rodríguez-Gonzálvez, P., Remondino, F., Minto, S., Orlandini, S., and Fuller, A., 2015. Accuracy evaluation of a mobile mapping system with advanced statistical methods, Int. Arch. Photogramm. Remote Sens. Spatial Inf. Sci., XL-5/W4, 245-253, https://doi.org/10.5194/isprsarchives-XL-5-W4-2452015. 